Pacific

Journal of

Mathematics

UNIMODAL SEQUENCES AND "STRANGE" FUNCTIONS:

A FAMILY OF QUANTUM MODULAR FORMS

Kathrin Bringmann, Amanda Folsom and Robert C. RhOAdes 


\title{
UNIMODAL SEQUENCES AND “STRANGE" FUNCTIONS: A FAMILY OF QUANTUM MODULAR FORMS
}

\author{
KATHRin Bringmann, Amanda Folsom And Robert C. RHOAdes
}

\begin{abstract}
We construct an infinite family of quantum modular forms from combinatorial rank "moment" generating functions for strongly unimodal sequences. The first member of this family is Kontsevich's "strange" function studied by Zagier. These results rely upon the theory of mock Jacobi forms. As a corollary, we exploit the quantum and mock modular properties of these combinatorial functions in order to obtain asymptotic expansions.
\end{abstract}

\section{Introduction and statement of results}

A sequence of integers $\left\{a_{j}\right\}_{j=1}^{s}$ is called a strongly unimodal sequence of size $n$ if there exists an integer $k$ such that

$$
0<a_{1}<a_{2}<\cdots<a_{k}>a_{k+1}>\cdots>a_{s}>0
$$

and $a_{1}+\cdots+a_{s}=n$. A number of familiar sequences are strongly unimodal, for example, the sequence of binomial coefficients $\left\{\left(\begin{array}{c}n \\ j-1\end{array}\right)\right\}_{j=1}^{n+1}$ with $n$ even. Attached to strongly unimodal sequences is a notion of rank, analogous to the well-known notion of the rank of an integer partition. For more on partition ranks, see for example original works in [Ramanujan 1919; Dyson 1944; Atkin and SwinnertonDyer 1954], and the more recent joint work of [Bringmann and Ono 2010] related to mock modular forms. The rank of a strongly unimodal sequence is equal to $s-2 k+1$, the number of terms after the maximal term minus the number of terms that precede it. For example, there are six strongly unimodal sequences of size 5: $\{5\}$, $\{1,4\},\{4,1\},\{1,3,1\},\{2,3\},\{3,2\}$. Their respective ranks are $0,-1,1,0,-1,1$. By letting $w$ (resp. $w^{-1}$ ) keep track of the terms after (resp. before) a maximal term, we have that $u(m, n)$, the number of size $n$ and rank $m$ sequences, satisfies

$$
U(w ; q):=\sum_{n=1}^{\infty} \sum_{m=-\infty}^{\infty} u(m, n)(-w)^{m} q^{n}=\sum_{n=0}^{\infty}(w q ; q)_{n}\left(w^{-1} q ; q\right)_{n} q^{n+1}
$$

MSC2010: primary 11F99; secondary 11F37, 33D15.

Keywords: quantum modular forms, mock modular forms, Jacobi forms, unimodal sequences, partitions, asymptotics, moment generating functions. 
where we set $(w ; q)_{n}:=\prod_{j=0}^{n-1}\left(1-w q^{j}\right)$, for $n \in \mathbb{N}_{0}$.

Recently, Bryson, Ono, Pitman, and the third author [Bryson et al. 2012] studied this function in the special case $w=1$, namely, ${ }^{1}$

$$
U(1 ; q)=\sum_{n=1}^{\infty} \sum_{m=-\infty}^{\infty}(-1)^{m} u(m, n) q^{n}=\sum_{n=1}^{\infty}\left(u_{e}(n)-u_{o}(n)\right) q^{n},
$$

where $u_{e}(n)$ (resp. $\left.u_{o}(n)\right)$ denotes the number of unimodal sequences of size $n$ with even (resp. odd) rank. They showed that for every root of unity $\zeta$,

$$
U(1 ; \zeta)=F\left(\zeta^{-1}\right)
$$

where Kontsevich's "strange" function is defined by

$$
F(q):=\sum_{n=0}^{\infty}(q ; q)_{n} .
$$

Previously, Zagier [2001] proved that this function satisfies the "identity"

$$
F(q)=-\frac{1}{2} \sum_{n=1}^{\infty} n\left(\frac{12}{n}\right) q^{\left(n^{2}-1\right) / 24}
$$

where $(\dot{)})$ is the Kronecker symbol. The two sides of (1-3) don't make sense simultaneously. Indeed, the right-hand side of (1-3) converges in the unit disk $|q|<1$, but nowhere on the unit circle. The identity (1-3) means that at roots of unity $\zeta, F(\zeta)$ (which is clearly a finite sum) agrees with the limit as $q$ approaches $\zeta$ radially within the unit disk of the function on the right-hand side of (1-3). Moreover, Zagier proved that for $x \in \mathbb{Q} \backslash\{0\}$,

$$
\phi(x)+(-i x)^{-3 / 2} \phi\left(-\frac{1}{x}\right)=\frac{\sqrt{3 i}}{2 \pi} \int_{0}^{i \infty}(w+x)^{-3 / 2} \eta(w) d w,
$$

where

$$
\phi(x):=e^{-\pi i x / 12} F\left(e^{-2 \pi i x}\right)
$$

and

$$
\eta(w):=e^{\pi i w / 12} \prod_{n=1}^{\infty}\left(1-e^{2 \pi i n w}\right)
$$

is the Dedekind eta function. Note that the constant $\sqrt{3 i} / 2 \pi$ in (1-4) is given explicitly in [Bryson et al. 2012]. There, the authors also gave a new proof of (1-4), using the fact that $U(1 ; q)$ is a (weak) mixed mock modular form for $|q|<1$. Here, we slightly modify the definition of "mixed mock modular form" given in

\footnotetext{
${ }^{1}$ Note that the function $U(w ; q)$, given in $(1-2)$, is equal to the function $U(-w ; q)$ as defined in
} [Bryson et al. 2012]. 
[Dabholkar et al. 2014] to mean functions that lie in the tensor product of the general spaces of mock modular forms and weakly holomorphic modular forms (up to possible rational multiples of $q$ powers). In particular, we do not require these functions to be holomorphic at the cusps, as in [loc. cit.]. Weak mixed mock modular forms in this sense occur in a variety of areas including combinatorics [Andrews 2005], algebraic geometry [Vafa and Witten 1994], Lie theory [Kac and Wakimoto 2001], Joyce invariants [Mellit and Okada 2009], and quantum black holes [Manschot 2011; Dabholkar et al. 2014].

The similarity between (1-4) and the usual modular transformation formula of a modular form in part motivated Zagier [2010] to introduce the notion of a quantum modular form. A quantum modular form of weight $k \in \frac{1}{2} \mathbb{Z}$ is a complex-valued function $f$ on $\mathbb{Q}$ such that for all $\gamma=\left(\begin{array}{ll}a & b \\ c & d\end{array}\right) \in \mathrm{SL}_{2}(\mathbb{Z})$, the complex-valued function $h_{\gamma}$ defined on $\mathbb{Q} \backslash \gamma^{-1}(\infty)$ by

$$
h_{\gamma}(x):=f(x)-\varepsilon(\gamma)(c x+d)^{-k} f\left(\frac{a x+b}{c x+d}\right)
$$

satisfies a "suitable" property of continuity or analyticity. The $\varepsilon(\gamma)$ in (1-5) are suitable complex numbers, such as those in the theory of half-integral weight modular forms when $k \in \frac{1}{2} \mathbb{Z} \backslash \mathbb{Z}$.

This paper gives an infinite family of quantum modular forms from the "moments" of the unimodal rank statistic. In general, such moment functions are of both number theoretic and combinatorial interest. For example, in their celebrated work, Atkin and Garvan [2003] discovered a partial differential equation relating the bivariate generating functions for the partition statistics rank and crank, leading to exact linear relations between rank and crank moments. Andrews [2007] provided a beautiful combinatorial interpretation of partition rank moments in terms of " $k$ marked Durfee symbols". Andrews [2008] also discovered a relationship between partition rank moments and the "smallest parts" partition statistic, which has led to further work by Garvan [2011], for example. In addition to intrinsic combinatorial interest, moment functions have been shown to satisfy modular properties. For example, works including [Bringmann et al. 2009; 2010; Alfes et al. 2011] exhibit relationships to weak Maass forms and mock theta functions.

To state our results, we define for $r \in \mathbb{N}_{0}$ the "weighted" moment functions

$$
\phi_{r}(\tau):=(\pi i)^{2 r+1} \sum_{n=1}^{\infty} \sum_{m \in \mathbb{Z}}(-1)^{m} u(m, n) Q_{r}\left(m^{2}, n-\frac{1}{24}\right) q^{n-\frac{1}{24}},
$$

where here and throughout we set $q:=e^{2 \pi i \tau}$ and

$$
Q_{r}(X, Y):=\sum_{\substack{0 \leq \mu \leq r \\ 0 \leq \ell \leq r-\mu}} c_{r}(\mu, \ell) X^{\ell} Y^{\mu} \in \mathbb{Q}[X, Y]
$$


the rational coefficients $c_{r}(\mu, \ell)$ being defined in (1-9). For example, the first few polynomials (normalized, with $Y \rightarrow Y-\frac{1}{24}$ ) are given by

$$
\begin{aligned}
Q_{0}\left(X, Y-\frac{1}{24}\right) & =-2, \\
Q_{1}\left(X, Y-\frac{1}{24}\right) & =-4(X+2 Y), \\
Q_{2}\left(X, Y-\frac{1}{24}\right) & =-\frac{4}{105}\left(10 X+35 X^{2}+6 Y+180 X Y+108 Y^{2}\right), \\
Q_{3}\left(X, Y-\frac{1}{24}\right) & =-\frac{4}{3465}\left(7 X+140 X^{2}+154 X^{3}+2 Y+420 X Y\right. \\
& \left.+1260 X^{2} Y+120 Y^{2}+2520 X Y^{2}+720 Y^{3}\right) .
\end{aligned}
$$

Note that in particular the first member of the family $\phi_{r}(\tau)$ is (up to a constant) the "strange" function studied by Zagier and Kontsevich discussed above. That is, $\phi_{0}(\tau)=-2 \pi i q^{-1 / 24} U(1 ; q)=-2 \pi i \phi(\tau)$. It is not difficult to see that the functions $\phi_{r}(\tau)$ may also be written in terms of the "twisted" unimodal moment functions $u_{r}$, defined for integers $r \geq 0$ by

$$
u_{r}(q):=\sum_{n=1}^{\infty} \sum_{m \in \mathbb{Z}}(-1)^{m} u(m, n) m^{r} q^{n} .
$$

The moments $\sum_{m} u(m, n) m^{r}$ of the unimodal rank statistic are analogous with the rank and crank partition moments, functions which have drawn wide combinatorial interest since Atkin and Garvan [2003] famously introduced them. There is a vast literature on such objects, including asymptotic questions and congruence properties. While the unimodal rank moments are exponentially large for even $r$ [Bringmann et al. $\geq 2015]$, it is surprising that the twisted moments $\sum_{m}(-1)^{m} u(m, n) m^{r}$, as a consequence of our results, are only polynomially large in $n$. We have chosen to handle the more complicated expressions $\sum_{m}(-1)^{m} u(m, n) Q_{r}\left(m^{2}, n-\frac{1}{24}\right)$ because the generating functions for these numbers have a fixed weight as modular objects as seen in Theorem 1.1, while the generating function for the twisted moments will have a mixed weight. To relate these generating functions $\phi_{r}(\tau)$ to the twisted unimodal moments $u_{r}(\tau)$, by symmetry, we note that $u_{2 r+1}(q)=0$ for integers $r \geq 0$. In particular, using (1-6), we find that

$$
\phi_{r}(\tau)=(\pi i)^{2 r+1} \sum_{\substack{0 \leq \mu \leq r \\ 0 \leq \ell \leq r-\mu}} \frac{c_{r}(\mu, \ell)}{(2 \pi i)^{\mu}} \cdot \frac{\partial^{\mu}}{\partial \tau^{\mu}}\left(u_{2 \ell}(q) q^{-\frac{1}{24}}\right),
$$

where we define

$$
c_{r}(\mu, \ell):=\frac{-2^{2 \ell+1} 6^{\mu} \Gamma\left(\frac{1}{2}+2 r-\mu\right)}{\Gamma\left(\frac{1}{2}+2 r\right) \mu !(2 \ell) !(2 r-2 \mu-2 \ell+1) !} \in \mathbb{Q} .
$$

The coefficients $c_{r}(\mu, \ell)$ are indeed in $\mathbb{Q}$, as it is well known for integers $k \in \mathbb{N}_{0}$, that $\Gamma\left(\frac{1}{2}+k\right) \in \sqrt{\pi} \cdot \mathbb{Q}$. The twisted moment functions also naturally extend the unimodal function $U(1 ; q)$ discussed above; namely, $u_{0}(q)=U(1 ; q)=-q^{1 / 24}(2 \pi i)^{-1} \phi_{0}(\tau)$. 
To state our first result, we define another polynomial

$$
P_{r}(X, Y):=\sum_{\substack{0 \leq N \leq r \\ 0 \leq M \leq 3 r}} b_{r}(N, M) X^{2 N+1} Y^{M},
$$

where the coefficients $b_{r}(N, M)$ are given explicitly in (3-13). Our first theorem establishes that the unimodal moment functions $\phi_{r}$ are quantum modular forms on $\mathbb{Q} \backslash\{0\}$, and that their transformation law also extends to $\mathbb{T}$. The function $\mathcal{H}_{r}$ below is defined in (3-14).

Theorem 1.1. Let $r \in \mathbb{N}_{0}$. If $\tau \in \mathbb{U} \cup \mathbb{Q} \backslash\{0\}$, we have

$$
\begin{aligned}
\phi_{r}(\tau)-(-i \tau)^{-3 / 2-2 r} \phi_{r}\left(-\frac{1}{\tau}\right) \\
=\int_{\mathbb{R}} P_{r}\left(w,(-i \tau)^{-1}\right) e^{\pi i \tau w^{2} / 3} \frac{\sinh \left(\frac{2 \pi w}{3}\right)}{\cosh (\pi w)} d w+\mathcal{H}_{r}(\tau),
\end{aligned}
$$

where $\mathcal{H}_{r}(\tau)=0$ for $\tau \in \mathbb{Q} \backslash\{0\}$. In particular, the functions $\phi_{r}$ are quantum modular forms.

Remarks. (1) The transformation law given in (1-11) in the case $\tau \in \mathbb{H}$ essentially establishes the mock modular properties of the unimodal rank moment functions $\phi_{r}(\tau)$.

(2) In the course of proving (1-11) in the case $\tau \in \mathbb{Q} \backslash\{0\}$, we show that for each integer $r \geq 0$, the function $\phi_{r}$ is defined for $\tau \in \mathbb{Q}$. Moreover, in Theorem 5.1 of Section 5, we pay special attention to the case $r=1$, and establish an explicit finite value for $\phi_{1}(h / k)(h, k \in \mathbb{Z})$ as the value of a polynomial in the root of unity $e^{2 \pi i h / k}$.

(3) Our functions naturally arise from mock Jacobi forms. It would be interesting to investigate whether a theory of quantum Jacobi forms could be developed that contains functions arising in this paper as special cases.

Our next theorem exploits the automorphic properties given in Theorem 1.1, and establishes the asymptotic behavior of the moment functions $u_{r}$. While such properties are of independent interest, we also point out that these functions are related to the quantum moment functions $\phi_{r}$ by (1-8). To describe their asymptotic behavior, we use the Bernoulli polynomials $B_{k}(x)$ and Euler polynomials $E_{k}(x)$, defined by the generating functions

$$
\frac{z e^{x z}}{e^{z}-1}=\sum_{k=0}^{\infty} B_{k}(x) \frac{z^{k}}{k !}
$$

and

$$
\frac{2 e^{x z}}{e^{z}+1}=\sum_{k=0}^{\infty} E_{k}(x) \frac{z^{k}}{k !} .
$$


Theorem 1.2. For nonnegative integers $r$, as $t \rightarrow 0^{+}$, we have

$$
\begin{array}{r}
e^{\pi t / 12} u_{2 r}\left(e^{-2 \pi t}\right) \\
=\frac{3^{2 r+1}}{2 r+1} \sum_{k=0}^{\infty} \frac{(3 \pi t)^{k}}{k !} \sum_{0 \leq n \leq r}\left(\begin{array}{c}
2 r+1 \\
2 n
\end{array}\right) 3^{-2 n} B_{2 n}\left(\frac{1}{2}\right) E_{2 r+1+2 k-2 n}\left(\frac{5}{6}\right),
\end{array}
$$

In particular, we have

$$
e^{\pi t / 12} u_{2 r}\left(e^{-2 \pi t}\right) \sim \frac{2 \cdot 6^{2 r}}{2 r+1}\left(B_{2 r+1}\left(\frac{2}{3}\right)+B_{2 r+1}\left(\frac{5}{6}\right)\right) .
$$

The paper is organized as follows. In Section 2 we provide relevant background information on modular forms, Jacobi forms, and mock Jacobi forms, as well as Bernoulli and Euler polynomials. In Section 3 we prove Theorem 1.1, and in Section 4 we establish Theorem 1.2. In Section 5 we pay special consideration to the moment function $\phi_{1}$.

\section{Preliminaries}

Here, we provide preliminary information on automorphic forms in Section 2A, and Bernoulli and Euler polynomials in Section 2B.

2A. Automorphic forms. In this section, we recall some fundamental properties of certain modular and (mock) Jacobi forms. We start with the well-known transformation law for the Dedekind $\eta$-function.

Lemma 2.1. For $\gamma=\left(\begin{array}{ll}a & b \\ c & d\end{array}\right) \in \mathrm{SL}_{2}(\mathbb{Z})$, we have

$$
\eta(\gamma \tau)=\chi(\gamma)(c \tau+d)^{1 / 2} \eta(\tau),
$$

where $\chi(\gamma)$ is a 24-th root of unity, which can be given explicitly in terms of Dedekind sums [Rademacher 1973]. In particular, we have

$$
\eta\left(-\frac{1}{\tau}\right)=\sqrt{-i \tau} \eta(\tau) .
$$

Here and throughout the square root is defined by the principal branch of the logarithm. Moreover, we require the usual Jacobi theta function, defined for $z \in \mathbb{C}$ and $\tau \in \mathbb{U}$ by

$$
\vartheta(z ; \tau):=\sum_{\nu \in \frac{1}{2}+\mathbb{Z}} e^{\pi i \nu^{2} \tau+2 \pi i v\left(z+\frac{1}{2}\right)} .
$$

This function is well known to satisfy the following transformation law [Rademacher 1973, (80.31) and (80.8)]: 
Lemma 2.2. For $\lambda, \mu \in \mathbb{Z}$ and $\gamma=\left(\begin{array}{ll}a & b \\ c & d\end{array}\right) \in \mathrm{SL}_{2}(\mathbb{Z})$, we have

$$
\begin{aligned}
& \vartheta(z+\lambda \tau+\mu ; \tau)=(-1)^{\lambda+\mu} q^{-\lambda^{2} / 2} e^{-2 \pi i \lambda z} \vartheta(z ; \tau), \\
& \vartheta\left(\frac{z}{c \tau+d} ; \gamma \tau\right)=\chi^{3}(\gamma)(c \tau+d)^{1 / 2} e^{\pi i c z^{2} /(c \tau+d)} \vartheta(z ; \tau) .
\end{aligned}
$$

In particular,

$$
\vartheta\left(\frac{z}{\tau} ;-\frac{1}{\tau}\right)=-i \sqrt{-i \tau} e^{\pi i z^{2} / \tau} \vartheta(z ; \tau) .
$$

The Jacobi theta function also satisfies the well-known triple product identity $\left(w=e^{2 \pi i z}\right)$

$$
\vartheta(z ; \tau)=-i q^{1 / 8} w^{-1 / 2} \prod_{n=1}^{\infty}\left(1-q^{n}\right)\left(1-w q^{n-1}\right)\left(1-w^{-1} q^{n}\right) .
$$

Additionally, we require the following classical Taylor expansion (see for example [Zagier 1991]):

$$
\vartheta(z ; \tau)=-2 \pi z \cdot \eta^{3}(\tau) \exp \left(-2 \sum_{k=1}^{\infty} G_{2 k}(\tau) \frac{(2 \pi i z)^{2 k}}{(2 k) !}\right) .
$$

Here for even integers $k \geq 2$, the Eisenstein series are defined by

$$
G_{k}(\tau):=-\frac{B_{k}}{2 k}+\sum_{n=1}^{\infty} \sigma_{k-1}(n) q^{n},
$$

where $\sigma_{\ell}(n):=\sum_{d \mid n} d^{\ell}$ and $B_{k}$ denotes the $k$-th Bernoulli number.

We also make use of Zwegers' functions $A_{\ell}\left(z_{1}, z_{2} ; \tau\right)$ [2010] (see also [Bringmann 2008; Andrews et al. 2013]), defined for $\ell \in \mathbb{N}, \tau \in \mathbb{H}, z_{2} \in \mathbb{C}$, and $z_{1} \in \mathbb{C} \backslash(\mathbb{Z} \tau+\mathbb{Z})$ by

$$
A_{\ell}\left(z_{1}, z_{2} ; \tau\right):=e^{\ell \pi i z_{1}} \sum_{n \in \mathbb{Z}} \frac{(-1)^{\ell n} q^{\ell n(n+1) / 2} e^{2 \pi i n z_{2}}}{1-q^{n} e^{2 \pi i z_{1}}} .
$$

These functions may be "completed" into nonholomorphic Jacobi forms by setting

$$
\widehat{A}_{\ell}\left(z_{1}, z_{2} ; \tau\right):=A_{\ell}\left(z_{1}, z_{2} ; \tau\right)+R_{\ell}\left(z_{1}, z_{2} ; \tau\right) .
$$

The nonholomorphic completions of these higher-level Appell functions are defined by

$R_{\ell}\left(z_{1}, z_{2} ; \tau\right):=\frac{i}{2} \sum_{k=0}^{\ell-1} e\left(k z_{1}\right) \vartheta\left(z_{2}+k \tau+\frac{\ell-1}{2} ; \ell \tau\right) R\left(\ell z_{1}-z_{2}-k \tau-\frac{\ell-1}{2} ; \ell \tau\right)$, where $e(x):=e^{2 \pi i x}$ and where (with $\tau=u+i v$ ) 


$$
R(z ; \tau):=\sum_{n \in \frac{1}{2}+\mathbb{Z}}\left(\operatorname{sgn}(n)-E\left(\left(n+\frac{\operatorname{Im}(z)}{v}\right) \sqrt{2 v}\right)\right)(-1)^{n-\frac{1}{2}} q^{-n^{2} / 2} e^{-2 \pi i n z}
$$

with $E(z):=2 \int_{0}^{z} e^{-\pi t^{2}} d t$. Proposition 2.3 below shows that the so-called "error to modularity" of the function $R(z ; \tau)$ is the Mordell integral, defined for $z \in \mathbb{C}$ and $\tau \in \mathbb{U}$ by

$$
h(z ; \tau):=\int_{\mathbb{R}} \frac{e^{\pi i \tau w^{2}-2 \pi z w}}{\cosh (\pi w)} d w .
$$

Proposition 2.3 [Zwegers 2002]. For $z \in \mathbb{C}$ and $\tau \in \mathbb{U}$, we have

$$
\begin{aligned}
& R(z+1 ; \tau)=-R(z ; \tau), \\
& R\left(\frac{z}{\tau} ;-\frac{1}{\tau}\right)=\sqrt{-i \tau} e^{-\pi i z^{2} / \tau}(-R(z ; \tau)+h(z ; \tau)) .
\end{aligned}
$$

The completed higher-level Appell functions $A_{\ell}\left(z_{1}, z_{2} ; \tau\right)$ transform as follows.

Proposition 2.4 [Zwegers 2010]. For $n_{1}, n_{2}, m_{1}, m_{2} \in \mathbb{Z}$ and $\gamma=\left(\begin{array}{ll}a & b \\ c & d\end{array}\right) \in \mathrm{SL}_{2}(\mathbb{Z})$, we have

$$
\begin{aligned}
& \widehat{A}_{\ell}\left(z_{1}+n_{1} \tau+m_{1}, z_{2}+n_{2} \tau+m_{2} ; \tau\right) \\
& =(-1)^{\ell\left(n_{1}+m_{1}\right)} e\left(z_{1}\left(\ell n_{1}-n_{2}\right)-n_{1} z_{2}\right) q^{\ell n_{1}^{2} / 2-n_{1} n_{2}} \widehat{A}_{\ell}\left(z_{1}, z_{2} ; \tau\right), \\
& \widehat{A}_{\ell}\left(\frac{z_{1}}{c \tau+d}, \frac{z_{2}}{c \tau+d} ; \gamma \tau\right)=(c \tau+d) e\left(\frac{c\left(-\ell z_{1}^{2}+2 z_{1} z_{2}\right)}{2(c \tau+d)}\right) \widehat{A}_{\ell}\left(z_{1}, z_{2} ; \tau\right) .
\end{aligned}
$$

We further require "dissection properties" of the functions $\vartheta$ and $R$ (see [Shimura 1973; Zwegers 2010; Bringmann and Folsom 2013]).

Lemma 2.5. With notation as above, we have for $n \in \mathbb{N}$,

$$
\begin{aligned}
& \vartheta\left(z ; \frac{\tau}{n}\right)=\sum_{\ell=0}^{n-1} q^{\left(\ell-\frac{n-1}{2}\right)^{2} /(2 n)} e^{2 \pi i\left(\ell-\frac{n-1}{2}\right)\left(z+\frac{1}{2}\right)} \vartheta\left(n z+\left(\ell-\frac{n-1}{2}\right) \tau+\frac{n-1}{2} ; n \tau\right), \\
& R\left(z ; \frac{\tau}{n}\right)=\sum_{\ell=0}^{n-1} q^{-\left(\ell-\frac{n-1}{2}\right)^{2} /(2 n)} e^{-2 \pi i\left(\ell-\frac{n-1}{2}\right)\left(z+\frac{1}{2}\right)} R\left(n z+\left(\ell-\frac{n-1}{2}\right) \tau+\frac{n-1}{2} ; n \tau\right) .
\end{aligned}
$$

2B. Bernoulli and Euler polynomials. In this section, we recall certain properties of the Bernoulli polynomials $B_{k}(x)$ and Euler polynomials $E_{k}(x)$, defined in (1-12) and (1-13), respectively, as well as their special values

$$
B_{k}:=B_{k}(0), \quad E_{k}:=2^{k} E_{k}\left(\frac{1}{2}\right) .
$$


One property we make use of is a "dissection" property of the Bernoulli polynomials (see [Abramowitz and Stegun 1964, Chapter 23])

$$
B_{k}(m x)=m^{k-1} \sum_{a=0}^{m-1} B_{k}\left(x+\frac{a}{m}\right) \quad \text { for } m \in 2 \mathbb{N}_{0}+1 .
$$

Another "splitting" property that we use is

$$
2^{k} B_{k}\left(\frac{x+y}{2}\right)=\sum_{j=0}^{k}\left(\begin{array}{l}
k \\
j
\end{array}\right) B_{j}(x) E_{k-j}(y),
$$

which follows easily from the definition of the Euler and Bernoulli polynomials, using the fact that

$$
\frac{2 z \cdot e^{(x+y) z}}{e^{2 z}-1}=\frac{z e^{x z}}{e^{z}-1} \cdot \frac{2 e^{y z}}{e^{z}+1}
$$

Here and throughout, we let $\zeta_{N}:=e^{2 \pi i / N}$ for $N \in \mathbb{N}$. The next lemma expresses derivatives of secant in terms of Euler polynomials.

Lemma 2.6. With notation as above, we have, for $c \in \mathbb{N}_{0}$,

$$
\sec ^{(2 c+1)}\left(\frac{\pi}{3}\right)=(-1)^{c} \sqrt{3} \cdot 6^{2 c+1} E_{2 c+1}\left(\frac{5}{6}\right) .
$$

Proof. This follows quickly from [Cvijović 2009, Theorem 2]. Namely, using the facts that $E_{2 c-1}\left(\frac{1}{6}\right)=-E_{2 c-1}\left(\frac{5}{6}\right)$ and $E_{2 c-1}\left(\frac{1}{2}\right)=0$ gives the claim.

A fourth property that we use expresses the Euler numbers as integrals. Namely, it is known (see [Erdélyi et al. 1981, p. 42, Equation (18)] for example) that for $k \in \mathbb{N}_{0}$,

$$
\int_{\mathbb{R}} \frac{w^{2 k}}{\cosh (\pi w)} d w=(2 i)^{-2 k} E_{2 k}
$$

Note that $E_{2 k-1}=0$ for $k \in \mathbb{N}$.

\section{Proof of Theorem 1.1}

Here, we ultimately conclude Theorem 1.1 from Propositions 3.6-3.8 below. In Section 3A, we establish properties of mock Jacobi forms related to the unimodal rank generating function; and in Section 3B, we construct mock modular forms from its Taylor coefficients. In Section 3C, we establish quantum modularity and prove Theorem 1.1. Until otherwise indicated, throughout this section, we take $\tau \in \mathbb{H}$. 
3A. Mock Jacobi forms and unimodal ranks. Here we establish properties of mock Jacobi forms associated to the unimodal rank generating function. We begin by writing $U(w ; q)$ in terms of the Appell functions $A_{\ell}(u, v ; \tau)$ defined in (2-4). Throughout, for $w_{1}, w_{2} \in \mathbb{C}$, we let

$$
\mathcal{U}\left(w_{1} ; w_{2}\right):=U\left(e\left(w_{1}\right) ; e\left(w_{2}\right)\right) .
$$

Lemma 3.1. Let $w=e(z)$. With notation as above, we have

$$
\mathcal{U}(z ; \tau)=\frac{1}{\left(w^{1 / 2}-w^{-1 / 2}\right)(q ; q)_{\infty}}\left(A_{1}(z,-z ; \tau)-w^{-1} A_{3}(z,-\tau ; \tau)\right) .
$$

Proof. Entry 3.4.7 of "Ramanujan's lost notebook" (see [Andrews and Berndt 2009, p. 67]) gives with $a=-w, b=-w^{-1}$ that $\mathcal{U}(z ; \tau)$ equals

$$
\begin{aligned}
& \frac{-1}{(1-w)\left(1-w^{-1}\right)} \sum_{n=0}^{\infty} \frac{q^{n^{2}}}{(w q ; q)_{n}\left(w^{-1} q ; q\right)_{n}} \\
& \quad+\frac{1}{\left(1-w^{-1}\right)(q ; q)_{\infty}} \sum_{n \in \mathbb{Z}} \frac{(-1)^{n} q^{n(n+1) / 2} w^{-n}}{1-w q^{n}} .
\end{aligned}
$$

We note that the second sum on the right-hand side of (3-1) is easily seen to equal

$$
\frac{1}{\left(w^{1 / 2}-w^{-1 / 2}\right)(q ; q)_{\infty}} A_{1}(z,-z ; \tau) .
$$

Using these facts, the result follows after applying the identity (see [Atkin and Swinnerton-Dyer 1954])

$$
\begin{aligned}
& \frac{-1}{\left(1-w^{-1}\right)(1-w)} \sum_{n=0}^{\infty} \frac{q^{n^{2}}}{(w q ; q)_{n}\left(w^{-1} q ; q\right)_{n}} \\
& \quad=\frac{-1}{\left(w^{1 / 2}-w^{-1 / 2}\right)} \frac{1}{(q ; q)_{\infty}} A_{3}(z,-\tau ; \tau) .
\end{aligned}
$$

Next we define a normalization of the function $\mathcal{U}(z ; \tau)$

$$
\begin{aligned}
Y^{+}(z ; \tau) & :=-\left(w^{1 / 2}-w^{-1 / 2}\right) q^{-1 / 24} \cdot \mathcal{U}(z ; \tau) \\
& =\eta^{-1}(\tau)\left(w^{-1} A_{3}(z,-\tau ; \tau)-A_{1}(z,-z ; \tau)\right),
\end{aligned}
$$

where the second equality follows from Lemma 3.1. Using Proposition 3.3, we now establish a transformation law for $Y^{+}$, which is a key step in showing quantum modularity of the functions $\phi_{r}$. To state this, we define

$$
H(z ; \tau):=\frac{i}{2} \frac{\vartheta(z ; \tau)}{\eta(\tau)} h(2 z ; \tau)-g(z ; \tau),
$$


where $h(z ; \tau)$ is given in $(2-5)$, and

$$
g(z ; \tau):=\frac{i}{\sqrt{3}} \int_{\mathbb{R}} e^{\pi i \tau w^{2} / 3-2 \pi w z} \frac{\sinh \left(\frac{2 \pi w}{3}\right)}{\cosh (\pi w)} d w .
$$

Proposition 3.2. With notation as above, we have

$$
-i e^{3 \pi i z^{2} / \tau} Y^{+}\left(\frac{z}{\tau} ;-\frac{1}{\tau}\right) \frac{1}{\sqrt{-i \tau}}-Y^{+}(z ; \tau)=H(z ; \tau) .
$$

To prove Proposition 3.2 we rather work with a second normalization of the function $\mathcal{U}(z ; \tau)$, namely,

$$
\begin{aligned}
X^{+}(z ; \tau) & :=-e^{-3 \pi z^{2} /(2 v)}\left(w^{1 / 2}-w^{-1 / 2}\right)(q ; q)_{\infty} \mathcal{U}(z ; \tau) \\
& =\left(w^{-1} A_{3}(z,-\tau ; \tau)-A_{1}(z,-z ; \tau)\right) e^{-3 \pi z^{2} /(2 v)} .
\end{aligned}
$$

Moreover we need the completed function

$$
\begin{aligned}
\widehat{X}(z ; \tau) & :=\left(w^{-1} \widehat{A}_{3}(z,-\tau ; \tau)-\widehat{A}_{1}(z,-z ; \tau)\right) e^{-3 \pi z^{2} /(2 v)} \\
& =\left(\widehat{A}_{3}(z, 0 ; \tau)-\widehat{A}_{1}(z,-z ; \tau)\right) e^{-3 \pi z^{2} /(2 v)},
\end{aligned}
$$

where the second equality follows from the first transformation in Proposition 2.4.

Using Proposition 2.4, it is not difficult to establish a modularity result for $\widehat{X}(z ; \tau)$ :

Proposition 3.3. With notation as above, for $\gamma=\left(\begin{array}{ll}a & b \\ c & d\end{array}\right) \in \mathrm{SL}_{2}(\mathbb{Z})$, we have

$$
\widehat{X}\left(\frac{z}{c \tau+d} ; \gamma \tau\right)=(c \tau+d) \widehat{X}(z ; \tau)
$$

From Proposition 3.3, we can establish a transformation property of $X^{+}(z ; \tau)$ :

Proposition 3.4. With notation as above, we have that

$$
\begin{aligned}
X^{+}\left(\frac{z}{\tau} ;-\frac{1}{\tau}\right) \tau^{-1} & -X^{+}(z ; \tau) \\
& =\left(\frac{i}{2} \vartheta(z ; \tau) h(2 z ; \tau)+\frac{i}{2 \sqrt{3}} \eta(\tau) \sum_{ \pm} \pm h\left(z \pm \frac{1}{3} ; \frac{\tau}{3}\right)\right) e^{-3 \pi z^{2} /(2 v)} .
\end{aligned}
$$

Proof. Using Proposition 3.3 we obtain that

$$
\left(X^{+}\left(\frac{z}{\tau} ;-\frac{1}{\tau}\right) \tau^{-1}-X^{+}(z ; \tau)\right) 2 i=f_{1}(z ; \tau)+f_{2}(z ; \tau),
$$


with

$$
\begin{aligned}
f_{1}(z ; \tau):=\vartheta\left(-\frac{1}{\tau} ;-\frac{3}{\tau}\right) e^{-3 \pi z^{2} \bar{\tau} /(2 v \tau)} \tau^{-1} \sum_{ \pm} \pm e^{ \pm 2 \pi i z / \tau} R\left(\frac{3 z}{\tau} \pm \frac{1}{\tau} ;-\frac{3}{\tau}\right) \\
-\vartheta(\tau ; 3 \tau) e^{-3 \pi z^{2} /(2 v)} \sum_{ \pm} \pm e^{ \pm 2 \pi i z} R(3 z \mp \tau ; 3 \tau),
\end{aligned}
$$

$f_{2}(z ; \tau):=\vartheta\left(\frac{z}{\tau} ;-\frac{1}{\tau}\right) R\left(\frac{2 z}{\tau} ;-\frac{1}{\tau}\right) e^{-3 \pi z^{2} \bar{\tau} /(2 v \tau)} \tau^{-1}-\vartheta(z ; \tau) R(2 z ; \tau) e^{-3 \pi z^{2} /(2 v)}$.

We next simplify $f_{1}$ and $f_{2}$. Firstly, using Lemma 2.2 and Proposition 2.3 , we obtain that

$$
f_{2}(z ; \tau)=-\vartheta(z ; \tau) h(2 z ; \tau) e^{-3 \pi z^{2} /(2 v)} .
$$

Next Lemma 2.2 and Proposition 2.3 yield that

$$
\begin{aligned}
\vartheta\left(-\frac{1}{\tau} ;-\frac{3}{\tau}\right) e^{-3 \pi z^{2} \bar{\tau} /(2 v \tau)} \tau^{-1} \sum_{ \pm} \pm e^{ \pm 2 \pi i z / \tau} R\left(\frac{3 z}{\tau} \pm \frac{1}{\tau} ;-\frac{3}{\tau}\right) \\
=-\frac{1}{3} e^{-3 \pi z^{2} /(2 v)} \vartheta\left(-\frac{1}{3} ; \frac{\tau}{3}\right) \sum_{ \pm} \pm\left(-R\left(z \pm \frac{1}{3} ; \frac{\tau}{3}\right)+h\left(z \pm \frac{1}{3} ; \frac{\tau}{3}\right)\right) .
\end{aligned}
$$

Now Lemma 2.5, the fact that $\vartheta(0 ; \tau)=0$, and Proposition 2.3, give that

$$
\begin{aligned}
\vartheta\left(-\frac{1}{3} ; \frac{\tau}{3}\right) & =2 i \sin \left(\frac{\pi}{3}\right) q^{1 / 6} \vartheta(\tau ; 3 \tau), \\
R\left(z \pm \frac{1}{3} ; \frac{\tau}{3}\right) & =-q^{-\frac{1}{6}} e^{2 \pi i\left(z \pm \frac{1}{3}\right)} R(3 z-\tau ; 3 \tau)+R(3 z ; 3 \tau) \\
& -q^{-1 / 6} e^{-2 \pi i\left(z \pm \frac{1}{3}\right)} R(3 z+\tau ; 3 \tau) .
\end{aligned}
$$

Thus

$$
\sum_{ \pm} \mp R\left(z \pm \frac{1}{3} ; \frac{\tau}{3}\right)=2 i \sin \left(\frac{2 \pi}{3}\right) q^{-1 / 6} \sum_{ \pm} \pm e^{ \pm 2 \pi i z} R(3 z \mp \tau ; 3 \tau),
$$

and hence

$$
f_{1}(z ; \tau)=-\frac{i}{\sqrt{3}} q^{1 / 6} \vartheta(\tau ; 3 \tau) \sum_{ \pm} \pm h\left(z \pm \frac{1}{3} ; \frac{\tau}{3}\right) e^{-3 \pi z^{2} /(2 v)} .
$$

Combining (3-4), (3-5), and the fact that $\vartheta(\tau ; 3 \tau)=-i q^{-1 / 6} \eta(\tau)$ gives the claim.

Proof of Proposition 3.2. First note that

$$
\sum_{ \pm} \pm h\left(z \pm \frac{1}{3} ; \frac{\tau}{3}\right)=2 i \sqrt{3} \cdot g(z ; \tau)
$$


The result now follows immediately from Proposition 3.4 and Lemma 2.1, using the fact that

$$
Y^{+}(z ; \tau)=\frac{e^{3 \pi z^{2} /(2 v)}}{\eta(\tau)} X^{+}(z ; \tau) .
$$

3B. Taylor coefficients and unimodal ranks. Using the results from Section 3A, we next construct mock modular forms from the Taylor coefficients of the unimodal rank generating function. The functions $H(z ; \tau)$ and $Y^{+}(z ; \tau)$ are holomorphic in $z$, and it is not difficult to see that they are both odd functions in $z$. So we may write

$$
\begin{aligned}
Y^{+}(z ; \tau) & =\sum_{r=0}^{\infty} a_{2 r}(\tau) z^{2 r+1}, \\
H(z ; \tau) & =\sum_{r=0}^{\infty} h_{2 r}(\tau) z^{2 r+1} .
\end{aligned}
$$

The next lemma describes the modularity properties of the Taylor coefficients $a_{2 r}(\tau)$ of $Y^{+}(z ; \tau)$.

Lemma 3.5. With notation as above, we have

$$
a_{2 r}\left(-\frac{1}{\tau}\right)(-i \tau)^{-3 / 2-2 r}=\sum_{0 \leq j \leq r} \frac{(3 \pi)^{r-j}}{(r-j) !}(-1)^{j+1}(-i \tau)^{j-r}\left(a_{2 j}(\tau)+h_{2 j}(\tau)\right) .
$$

Proof. Proposition 3.2 directly yields

$$
Y^{+}\left(\frac{z}{\tau} ;-\frac{1}{\tau}\right)=i e^{-3 \pi i z^{2} / \tau} \sqrt{-i \tau}\left(Y^{+}(z ; \tau)+H(z ; \tau)\right) .
$$

Inserting (3-6), (3-7), and the Taylor expansion of the exponential function, we obtain

$$
\begin{aligned}
\sum_{r=0}^{\infty} a_{2 r}( & \left.-\frac{1}{\tau}\right)\left(\frac{z}{\tau}\right)^{2 r+1} \\
& =i \sqrt{-i \tau} \sum_{\ell=0}^{\infty} \frac{\left(-3 \pi i z^{2} / \tau\right)^{\ell}}{\ell !} \sum_{j=0}^{\infty}\left(a_{2 j}(\tau)+h_{2 j}(\tau)\right) z^{2 j+1} \\
& =i \sqrt{-i \tau} \sum_{r=0}^{\infty} z^{2 r+1} \sum_{0 \leq j \leq r} \frac{(3 \pi)^{r-j}}{(r-j) !}(-1)^{r+j}(-i \tau)^{j-r}\left(a_{2 j}(\tau)+h_{2 j}(\tau)\right) .
\end{aligned}
$$

Equating the coefficients of $z^{2 r+1}$ gives the claim.

To prove the transformation law for the functions $\phi_{r}$, we define for $r \in \mathbb{N}_{0}$,

$$
b_{2 r}(\tau):=\sum_{0 \leq \mu \leq r} \frac{(3 \pi i)^{\mu} \Gamma\left(\frac{1}{2}+2 r-\mu\right)}{\Gamma\left(\frac{1}{2}+2 r\right) \mu !} a_{2 r-2 \mu}^{(\mu)}(\tau) .
$$


We will later show that $\phi_{r}(\tau)=b_{2 r}(\tau)$. The functions $b_{2 r}(\tau)$ transform as described in the following proposition, a fact which follows as in [Eichler and Zagier 1985], using Lemma 3.5.

Proposition 3.6. With notation as above, for $r \in \mathbb{N}_{0}$, we have

$$
\begin{aligned}
& b_{2 r}\left(-\frac{1}{\tau}\right)(-i \tau)^{-3 / 2-2 r}- b_{2 r}(\tau) \\
&=-(-i \tau)^{-3 / 2-2 r} \sum_{0 \leq \mu \leq r} \frac{(3 \pi i)^{\mu} \Gamma\left(\frac{1}{2}+2 r-\mu\right)}{\Gamma\left(\frac{1}{2}+2 r\right) \mu !} \\
& \times \sum_{0 \leq j \leq r-\mu} \frac{(3 \pi)^{r-\mu-j}(-1)^{j}}{(r-\mu-j) !} \frac{\partial^{\mu}}{\partial \tau^{\mu}}\left((-i \tau)^{j+r-\mu+\frac{3}{2}} h_{2 j}(\tau)\right) .
\end{aligned}
$$

Our next proposition shows that the "errors to modularity" $h_{2 r}$ are $C^{\infty}$, a fact we use in the course of establishing the quantum modularity of the unimodal rank functions $\phi_{r}$ in Theorem 1.1. In doing so, we split the Taylor expansion of $H(z ; \tau)$ into two pieces

$$
H(z ; \tau)=H_{1}(z ; \tau)+H_{2}(z ; \tau),
$$

with

$$
\begin{aligned}
& H_{1}(z ; \tau)=\sum_{r=0}^{\infty} h_{1,2 r}(\tau) z^{2 r+1}:=\frac{i}{2} \frac{\vartheta(z ; \tau)}{\eta(\tau)} h(2 z ; \tau), \\
& H_{2}(z ; \tau)=\sum_{r=0}^{\infty} h_{2,2 r}(\tau) z^{2 r+1}:=-g(z ; \tau) .
\end{aligned}
$$

Proposition 3.7. The functions $h_{2 r}$ are $C^{\infty}$ on $\mathbb{R}$. To be more precise, $h_{1,2 r}(\tau)$ vanishes to infinite order for $\tau \in \mathbb{Q}$, and we extend this function to equal 0 on all of $\mathbb{R}$. Moreover, for $\tau \in \mathbb{U} \cup \mathbb{Q}$, the function $h_{2,2 r}$ satisfies

$$
h_{2,2 r}(\tau)=\frac{i}{\sqrt{3}} \frac{(2 \pi)^{2 r+1}}{(2 r+1) !} \int_{\mathbb{R}} e^{\pi i \tau w^{2} / 3} w^{2 r+1} \frac{\sinh \left(\frac{2 \pi w}{3}\right)}{\sinh (\pi w)} d w .
$$

Proof. Firstly, we have

$$
\begin{aligned}
& H_{1}(z ; \tau) \\
& \quad=\frac{i}{2 \eta(\tau)} \sum_{r=0}^{\infty} \frac{\partial^{r}}{\partial z^{r}}[\vartheta(z ; \tau) h(2 z ; \tau)]_{z=0} \frac{z^{r}}{r !} \\
& =\frac{i}{2 \eta(\tau)} \sum_{r=0}^{\infty} \frac{z^{2 r+1}}{(2 r+1) !} \sum_{\ell=0}^{r}\left(\begin{array}{l}
2 r+1 \\
2 \ell+1
\end{array}\right) \frac{\partial^{2 \ell+1}}{\partial z^{2 \ell+1}}[\vartheta(z ; \tau)]_{z=0} \frac{\partial^{2 r-2 \ell}}{\partial z^{2 r-2 \ell}}[h(2 z ; \tau)]_{z=0},
\end{aligned}
$$


so that

$h_{1,2 r}(\tau)=\frac{i}{2 \eta(\tau)} \sum_{\ell=0}^{r} \frac{1}{(2 \ell+1) !(2 r-2 \ell) !} \frac{\partial^{2 \ell+1}}{\partial z^{2 \ell+1}}[\vartheta(z ; \tau)]_{z=0} \frac{\partial^{2 r-2 \ell}}{\partial z^{2 r-2 \ell}}[h(2 z ; \tau)]_{z=0}$.

It is not hard to see that $h(2 z ; \tau)$ is $C^{\infty}$ as a function of $\tau$ near $z=0$. Moreover by (2-3), we see that

$$
\frac{i}{2 \eta(\tau)} \frac{\partial^{2 \ell+1}}{\partial z^{2 \ell+1}}[\vartheta(z ; \tau)]_{z=0}
$$

gives a linear combination of Eisenstein series multiplied by $\eta^{2}(\tau)$. It is well known that the Eisenstein series satisfy

$$
G_{k}\left(-\frac{1}{\tau}\right)=\tau^{k} G_{k}(\tau) \quad(k>2, \text { even })
$$

and

$$
G_{2}\left(-\frac{1}{\tau}\right)=\tau^{2} G_{2}(\tau)+\frac{i \tau}{4 \pi}
$$

This implies that the function $h_{2 r}(\tau)$ and its derivatives vanish exponentially for $\tau \in \mathbb{Q}$. The second claim follows directly by inserting the Taylor expansion of $e^{-2 \pi z x}$.

3C. Quantum unimodal ranks. Building from the results in Sections 3A and 3B, here we prove Theorem 1.1.

Proof of Theorem 1.1. We first relate the Taylor coefficients of $Y^{+}(z ; \tau)$ to the unimodal moments $u_{2 r}$. Using the definition of $u_{2 r}$, it is not difficult to verify that

$$
\mathcal{U}(z ; \tau)=\sum_{r=0}^{\infty} u_{2 r}(q) \frac{(2 \pi i z)^{2 r}}{(2 r) !}
$$

Using the Taylor expansion of $\sin (\pi z)$ we find that

$$
\begin{aligned}
Y^{+}(z ; \tau) & =-2 i q^{-1 / 24} \sin (\pi z) \mathcal{U}(z ; \tau) \\
& =-(2 \pi i z) \sum_{r=0}^{\infty}(2 \pi i z)^{2 r} \sum_{0 \leq \ell \leq r} \frac{u_{2 \ell}(q) q^{-1 / 24} 2^{2 \ell-2 r}}{(2 \ell) !(2 r-2 \ell+1) !}
\end{aligned}
$$

yielding

$$
\frac{a_{2 r}(\tau)}{(2 \pi i)^{2 r+1}}=-\sum_{0 \leq \ell \leq r} \frac{u_{2 \ell}(q) q^{-1 / 24} 2^{2 \ell-2 r}}{(2 \ell) !(2 r-2 \ell+1) !}
$$

Using (3-11), the definition of $\phi_{r}(\tau)$ in (1-6), or its equivalent formulation given in (1-8), as well as the definition of $b_{2 r}(\tau)$ in (3-8), it is not difficult to see that for 
each $r \in \mathbb{N}_{0}, b_{2 r}(\tau)=\phi_{r}(\tau)$. Combining this with the fact that

$$
h_{2 j}(\tau)=h_{1,2 j}(\tau)+h_{2,2 j}(\tau),
$$

Proposition 3.6 yields

$$
\begin{aligned}
& \phi_{r}\left(-\frac{1}{\tau}\right)(-i \tau)^{-3 / 2-2 r}-\phi_{r}(\tau) \\
& =-(-i \tau)^{-3 / 2-2 r} \sum_{\substack{0 \leq \mu \leq r \\
0 \leq j \leq r-\mu}} \frac{(3 \pi)^{r-j}(-1)^{j} i^{\mu} \Gamma\left(\frac{1}{2}+2 r-\mu\right)}{\Gamma\left(\frac{1}{2}+2 r\right) \mu !(r-\mu-j) !} \\
& \times \frac{\partial^{\mu}}{\partial \tau^{\mu}}\left((-i \tau)^{j+r-\mu+\frac{3}{2}}\left(h_{1,2 j}(\tau)+h_{2,2 j}(\tau)\right)\right) .
\end{aligned}
$$

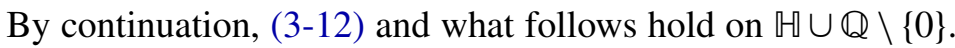

We first consider the first summand. We have by Proposition 3.7

$$
\begin{aligned}
& \frac{\partial^{\mu}}{\partial \tau^{\mu}}\left((-i \tau)^{j+r-\mu+\frac{3}{2}} h_{2,2 j}(\tau)\right) \\
& =\frac{i}{\sqrt{3}} \frac{(2 \pi)^{2 j+1}}{(2 j+1) !} \\
& \quad \times \int_{\mathbb{R}} \sum_{\ell=0}^{\mu}\left(\begin{array}{l}
\mu \\
\ell
\end{array}\right) \frac{\partial^{\ell}}{\partial \tau^{\ell}}\left((-i \tau)^{j+r-\mu+\frac{3}{2}}\right) \frac{\partial^{\mu-\ell}}{\partial \tau^{\mu-\ell}}\left(e^{\pi i w^{2} \tau / 3}\right) w^{2 j+1} \frac{\sinh \left(\frac{2 \pi w}{3}\right)}{\cosh (\pi w)} d w \\
& =\sum_{\ell=0}^{\mu}(-1)^{\ell} i^{\mu+1} \pi^{2 j+1+\mu-\ell} 2^{2 j+1} 3^{\ell-\mu-\frac{1}{2}}\left(\begin{array}{c}
\mu \\
\ell
\end{array}\right) \frac{\Gamma\left(j+r-\mu+\frac{5}{2}\right)}{(2 j+1) ! \Gamma\left(j+r-\mu+\frac{5}{2}-\ell\right)} \\
& \quad \times(-i \tau)^{j+r+\frac{3}{2}-\mu-\ell} \int_{\mathbb{R}} w^{2 j+2 \mu-2 \ell+1} e^{\pi i w^{2} \tau / 3} \frac{\sinh \left(\frac{2 \pi w}{3}\right)}{\cosh (\pi w)} d w .
\end{aligned}
$$

We now define the numbers

$$
\begin{aligned}
& b_{r}(\mu, j, \ell):= \\
& \quad \frac{i(-1)^{j+\ell+\mu} 2^{2 j+1} \pi^{r+j+\mu+1-\ell} 3^{r+\ell-\mu-j-\frac{1}{2}} \Gamma\left(\frac{1}{2}+2 r-\mu\right) \Gamma\left(j+r-\mu+\frac{5}{2}\right)}{(2 j+1) ! \ell !(\mu-\ell) !(r-\mu-j) ! \Gamma\left(\frac{1}{2}+2 r\right) \Gamma\left(j+r-\mu+\frac{5}{2}-\ell\right)},
\end{aligned}
$$

and let

$$
b_{r}(N, M):=\sum_{0 \leq \mu \leq r} \sum_{\begin{array}{c}
0 \leq j \leq r-\mu \\
0 \leq \ell \leq \mu \\
N=j+\mu-\ell \\
M=\mu+\ell+r-j
\end{array}} b_{r}(\mu, j, \ell)
$$


Moreover, we define $\mathcal{H}_{r}(\tau)$ to be

$$
\times \sum_{\substack{0 \leq \mu \leq r \\ 0 \leq j \leq r-\mu}}^{(-i \tau)^{-\frac{3}{2}-2 r}} \frac{(3 \pi)^{r-j}(-1)^{j} i^{\mu} \Gamma\left(\frac{1}{2}+2 r-\mu\right)}{\Gamma\left(\frac{1}{2}+2 r\right) \mu !(r-\mu-j) !} \frac{\partial^{\mu}}{\partial \tau^{\mu}}\left((-i \tau)^{j+r-\mu+\frac{3}{2}} h_{1,2 j}(\tau)\right) .
$$

Note that $\mathcal{H}_{r}(\tau)=0$ for $\tau \in \mathbb{Q} \backslash\{0\}$. We have thus shown for $\tau \in \mathbb{U} \cup \mathbb{Q} \backslash\{0\}$,

$$
\begin{aligned}
(-i \tau)^{-\frac{3}{2}-2 r} \phi_{r}\left(-\frac{1}{\tau}\right)-\phi_{r} & \\
& =-\int_{\mathbb{R}} P_{r}\left(w,(-i \tau)^{-1}\right) e^{\pi i \tau w^{2} / 3} \frac{\sinh \left(\frac{2 \pi w}{3}\right)}{\cosh (\pi w)} d w-\mathcal{H}_{r}(\tau),
\end{aligned}
$$

as claimed in (1-11).

Finally, under the translation $\tau \rightarrow \tau+1$, it is clear using the definition of $\phi_{r}(\tau)$ in (1-6) that $\phi_{r}(\tau+1)=e^{-\pi i / 12} \phi_{r}(\tau)$. With the proof of Proposition 3.8 below, using (1-8), Theorem 1.1 now follows.

We are left to show the existence of the moment functions and their derivatives.

Proposition 3.8. For $r, n \in \mathbb{N}_{0}$, the moment functions

$$
\frac{\partial^{n}}{\partial \tau^{n}}\left[q^{-1 / 24} u_{2 r}(q)\right]
$$

are defined for every root of unity $q=\zeta$ and lie in $\mathbb{Z}[\zeta]$.

Proof. For ease of notation, we let

$$
\begin{aligned}
D_{\alpha} & :=\alpha \frac{\partial}{\partial \alpha}, \\
J_{m}(w ; q) & :=(w q ; q)_{m}\left(w^{-1} q ; q\right)_{m} .
\end{aligned}
$$

To finish the proof it is enough to show that for $m$ sufficiently large, and every $n, r \in \mathbb{N}_{0}$, the function

$$
D_{q}^{n}\left(D_{w}^{r}\left[J_{m}(w ; q)\right]_{w=1}\right)
$$

vanishes for $q=\zeta$.

It is not difficult to see that for $m \in \mathbb{N}$,

$$
\frac{D_{w}\left(J_{m}(w ; q)\right)}{J_{m}(w ; q)}=-\sum_{k=1}^{m} \frac{w q^{k}}{1-w q^{k}}+\sum_{k=1}^{m} \frac{w^{-1} q^{k}}{1-w^{-1} q^{k}}=: R_{m}(w ; q) .
$$


We further relax notation and let $J:=J_{m}(w ; q), R:=R_{m}(w ; q)$, and $R^{(r)}:=D_{w}^{r} R$ for $r \in \mathbb{N}_{0}$. Using (3-16), we find that

$$
\begin{aligned}
& D_{w} J=J R, \\
& D_{w}^{2} J=J\left(R^{2}+R^{(1)}\right), \\
& D_{w}^{3} J=J\left(R^{3}+3 R R^{(1)}+R^{(2)}\right), \\
& D_{w}^{4} J=J\left(R^{4}+4 R R^{(2)}+3\left(R^{(1)}\right)^{2}+6 R^{2} R^{(1)}+R^{(3)}\right),
\end{aligned}
$$

Note that each $D_{w}^{r} J$ can be expressed as $J$ multiplied by a sum over the partitions of $r$. That is, given a partition $\pi=\ell_{1}(\pi) \cdot 1+\ell_{2}(\pi) \cdot 2+\cdots+\ell_{r-1}(\pi) \cdot(r-1)+\ell_{r}(\pi) \cdot r$ of $r$ (where each $\ell_{j}(\pi) \in \mathbb{N}_{0}$ ), we may assign the product

$$
\prod_{1 \leq j \leq r}\left(D_{w}^{j-1} R\right)^{\ell_{j}(\pi)} .
$$

Conversely, every such product appearing as a summand as above for $D_{w}^{r} J$ corresponds to a partition of $r$. In general, we have

$$
D_{w}^{r}\left[J_{m}(w ; q)\right]_{w=1}=(q ; q)_{m}^{2} \sum_{\pi \vdash r} c(\pi) \prod_{1 \leq j \leq r}\left(D_{w}^{j-1}\left[R_{m}(w ; q)\right]_{w=1}\right)^{\ell_{j}(\pi)},
$$

where we sum over all partitions $\pi$ of $r$. The exponents $\ell_{j}(\pi)$ correspond to the number of parts of the partition $\pi$ of $r$, and the constants $c(\pi)=c_{r}(\pi)$ also depend on the partition $\pi$ of $r$. Now using the definition of $R_{m}(w ; q)$ in (3-16), we may write

$$
\begin{aligned}
\sum_{\pi \vdash r} c(\pi) \prod_{1 \leq j \leq r}\left(D_{w}^{j-1}\left[R_{m}(w ; q)\right]_{w=1}\right)^{\ell_{j}(\pi)} & \\
& =\sum_{\vec{k}=\left(k_{1}, \ldots, k_{c}\right)} \frac{P_{\vec{k}, r}(q)}{\prod_{j=1}^{c}\left(1-q^{k_{j}}\right)^{r}}=: R_{m, r}(q),
\end{aligned}
$$

where $c=c_{r} \in \mathbb{N}$ depends only on $r$, and $P_{\vec{k}, r} \in \mathbb{Z}[q]$. Next we apply the operator $D_{q}^{n}$ to $(q ; q)_{m}^{2}$ multiplied by $R_{m, r}(q)$ in (3-17) above. Using the product rule, we have (3-15) equals

$$
\sum_{0 \leq j \leq n}\left(\begin{array}{c}
n \\
j
\end{array}\right) D_{q}^{j}\left((q ; q)_{m}^{2}\right) D_{q}^{n-j}\left(R_{m, r}(q)\right)
$$

It is not difficult to see that

$$
\frac{D_{q}\left((q ; q)_{m}^{2}\right)}{(q ; q)_{m}^{2}}=-2 \sum_{k=1}^{m} \frac{k q^{k}}{1-q^{k}}=: T_{m}(q),
$$


and for $l \in \mathbb{N}$, that

$$
D_{q}^{\ell-1}\left(T_{m}(q)\right)=\sum_{k=1}^{m} \frac{Q_{k, l}(q)}{\left(1-q^{k}\right)^{\ell}}
$$

with $Q_{k, l}(q) \in \mathbb{Z}[q]$. Therefore, we may conclude that (3-15) has the shape

$$
(q ; q)_{m}^{2} \sum_{\vec{k}=\left(k_{1}, \ldots, k_{d}\right)} \frac{P_{\vec{k}, r, n}(q)}{\prod_{j=1}^{d}\left(1-q^{k_{j}}\right)^{r+n}},
$$

where $d=d_{r, n} \in \mathbb{N}$ depends only on $r$ and $n$, and $P_{\vec{k}, r, n} \in \mathbb{Z}[q]$. Now if $\zeta=\zeta_{m}$ then $(q ; q)_{M}^{2}(M \in \mathbb{N})$ vanishes at $q=\zeta$ of order $\geq 2\lfloor m / M\rfloor$. On the other hand, each term

$$
\frac{P_{\vec{k}, r, n}(q)}{\prod_{j=1}^{d}\left(1-q^{k_{j}}\right)^{r+n}}
$$

vanishes at $q=\zeta$ of order at most $d(r+n)$, which is a constant independent of $m$. Thus, the claim follows.

\section{Proof of Theorem 1.2}

To prove Theorem 1.2, we recall (3-2). It is not difficult to see from Proposition 3.2 that

$$
Y^{+}(z ; i t)=-H(z ; i t)+\sum_{r \geq 0} \beta_{r}(t) z^{r}
$$

with

$$
\beta_{r}(t) \ll_{r} e^{-N / t}
$$

for some $N>0$. To find the asymptotic expansion of $H(z ; i t)$, we split as in (3-9) and bound using (2-3)

$$
h_{1,2 r}(i t) \ll e^{-M / t}
$$

for some $M>0$. Thus we are left to determine the asymptotic expansion of $H_{2}(z ; i t)$. For this, we write

$$
\begin{aligned}
H_{2}(z ; i t) & =-\frac{i}{\sqrt{3}} \int_{\mathbb{R}} e^{-\pi t w^{2} / 3-2 \pi w z} \frac{\sinh \left(\frac{2 \pi w}{3}\right)}{\cosh (\pi w)} d w \\
4-1) \quad & =-\frac{i}{\sqrt{3}} \sum_{r=0}^{\infty} \frac{(-2 \pi z)^{2 r+1}}{(2 r+1) !} \sum_{k=0}^{\infty} \frac{(-\pi t / 3)^{k}}{k !} \int_{\mathbb{R}} \frac{w^{2 r+2 k+1} \sinh \left(\frac{2 \pi w}{3}\right)}{\cosh (\pi w)} d w,
\end{aligned}
$$


where the identity in (4-1) refers to an asymptotic expansion. Thus, to determine the asymptotic expansion of $H_{2}(z ; i t)$, we are left to evaluate explicitly for $a \in \mathbb{N}_{0}$,

$$
\begin{aligned}
\mathcal{C}_{a} & :=\int_{\mathbb{R}} \frac{w^{2 a+1} \sinh \left(\frac{2 \pi w}{3}\right)}{\cosh (\pi w)} d w \\
& =\frac{1}{2} \int_{\mathbb{R}} \frac{w^{2 a+1}\left(e^{2 \pi w / 3}-e^{-2 \pi w / 3}\right)}{\cosh (\pi w)} d w=\sum_{r=1}^{\infty} \frac{\left(\frac{2 \pi}{3}\right)^{2 r-1}}{(2 r-1) !} \int_{\mathbb{R}} \frac{w^{2 a+2 r}}{\cosh (\pi w)} d w .
\end{aligned}
$$

From (2-8), we have that the integral above equals (2i) ${ }^{-2 a-2 r} E_{2 a+2 r}$, yielding

$$
\mathcal{C}_{a}=(-2 i)^{-2 a-1} \sum_{r=1}^{\infty} \frac{\left(\frac{\pi i}{3}\right)^{2 r-1}}{(2 r-1) !} E_{2 a+2 r}=(-2 i)^{-2 a-1} \sum_{r=0}^{\infty} \frac{\left(\frac{\pi i}{3}\right)^{r}}{r !} E_{2 a+r+1} .
$$

The second equality above holds because $E_{j}=0$ for $j$ odd.

We are thus left to understand $\sum_{r=0}^{\infty}\left(v^{r} / r !\right) E_{r+b}$ for positive integers $b$ and $v=\pi i / 3$. Set

$$
f(v):=\sum_{r=0}^{\infty} \frac{E_{r}}{r !} v^{r}=\operatorname{sech}(v),
$$

where the second equality above is simply the definition of the Euler numbers. Then

$$
f^{(b)}(v)=\sum_{r=0}^{\infty} \frac{E_{r+b}}{r !} v^{r}
$$

Thus

$$
\mathcal{C}_{a}=(-2 i)^{-2 a-1} \operatorname{sech}^{(2 a+1)}\left(\frac{\pi i}{3}\right)=2^{-2 a-1} \sec ^{(2 a+1)}\left(\frac{\pi}{3}\right) .
$$

Next we deduce from (1-12) that

$$
\frac{i}{2} \frac{1}{\sin (\pi z)}=-\sum_{n=0}^{\infty} \frac{B_{2 n}\left(\frac{1}{2}\right)}{(2 n) !}(2 \pi i z)^{2 n-1} .
$$

Combining the above, we have established that the asymptotic expansion of $\mathcal{U}(z ; i t) e^{\pi t / 12}$ as $t \rightarrow 0^{+}$is given by

$$
\frac{1}{\sqrt{3}} \sum_{r=0}^{\infty}(2 \pi i z)^{2 r}(-1)^{r} \sum_{0 \leq n \leq r} \frac{B_{2 n}\left(\frac{1}{2}\right)}{(2 n) !} \frac{(-1)^{n}}{(2 r-2 n+1) !} \sum_{k=0}^{\infty} \frac{(-\pi t / 3)^{k}}{k !} \mathcal{C}_{r-n+k} .
$$

Thus, using (4-2), we have the asymptotic expansion as $t \rightarrow 0^{+}$,

$$
\begin{aligned}
e^{\pi t / 12} u_{2 r}\left(e^{-2 \pi t}\right)=\frac{(2 r) !(-1)^{r} 2^{-2 r-1}}{\sqrt{3}} \sum_{k=0}^{\infty} \frac{t^{k}}{k !}\left(-\frac{\pi}{3}\right)^{k} 2^{-2 k} \\
\quad \times \sum_{0 \leq n \leq r} \frac{(-1)^{n} B_{2 n}\left(\frac{1}{2}\right) 2^{2 n}}{(2 n) !(2 r-2 n+1) !} \sec ^{(2 r-2 n+2 k+1)}\left(\frac{\pi}{3}\right) .
\end{aligned}
$$


Using Lemma 2.6 together with (4-3), we have

$$
\begin{aligned}
& e^{\pi t / 12} u_{2 r}\left(e^{-2 \pi t}\right) \\
& \quad=\frac{3^{2 r+1}}{2 r+1} \sum_{k=0}^{\infty} \frac{(3 \pi t)^{k}}{k !} \sum_{0 \leq n \leq r}\left(\begin{array}{c}
2 r+1 \\
2 n
\end{array}\right) 3^{-2 n} B_{2 n}\left(\frac{1}{2}\right) E_{2 r+2 k+1-2 n}\left(\frac{5}{6}\right),
\end{aligned}
$$

which concludes the proof of the first statement of Theorem 1.2.

Next we prove the claimed asymptotic for the main term. Since $B_{2 n+1}\left(\frac{1}{2}\right)=0$, we may rewrite the $k=0$ summand of (4-4) as

$$
\frac{3^{2 r+1}}{2 r+1} \sum_{0 \leq n \leq 2 r+1}\left(\begin{array}{c}
2 r+1 \\
n
\end{array}\right) 3^{-n} B_{n}\left(\frac{1}{2}\right) E_{2 r+1-n}\left(\frac{5}{6}\right) .
$$

Now we use (2-6), which yields that

$$
B_{n}\left(\frac{1}{2}\right)=3^{n-1} \sum_{a=0}^{2} B_{n}\left(\frac{1}{6}+\frac{a}{3}\right) .
$$

Thus, (4-5) equals

$$
\frac{3^{2 r}}{2 r+1} \sum_{a=0}^{2} \sum_{0 \leq n \leq 2 r+1}\left(\begin{array}{c}
2 r+1 \\
n
\end{array}\right) B_{n}\left(\frac{1}{6}+\frac{a}{3}\right) E_{2 r+1-n}\left(\frac{5}{6}\right) .
$$

Using (2-7), (4-6) reduces to

$$
\frac{2 \cdot 6^{2 r}}{2 r+1} \sum_{a=0}^{2} B_{2 r+1}\left(\frac{1}{2}+\frac{a}{6}\right)
$$

Noting again that $B_{2 r+1}\left(\frac{1}{2}\right)=0$, we find that as claimed, as $t \rightarrow 0^{+}$,

$$
e^{\pi t / 12} u_{2 r}\left(e^{-2 \pi t}\right) \sim \frac{2 \cdot 6^{2 r}}{2 r+1}\left(B_{2 r+1}\left(\frac{2}{3}\right)+B_{2 r+1}\left(\frac{5}{6}\right)\right) .
$$

\section{An example: the moment function $\phi_{1}(\tau)$}

In this section, we give an exact value for the quantum moment function

$$
\begin{aligned}
\phi_{1}(\tau) & =4 \pi^{3} i q^{-1 / 24} \sum_{n=1}^{\infty} \sum_{m \in \mathbb{Z}}(-1)^{m} u(m, n)\left(m^{2}+2 n\right) q^{n} \\
& =4 \pi^{3} i q^{-1 / 24}\left(u_{2}(q)-i \pi^{-1} \frac{\partial}{\partial \tau} u_{0}(q)\right) .
\end{aligned}
$$


To describe this, we define for positive integers $n$ the polynomials

$$
d_{n}(q):=n(q ; q)_{n-1}^{2} q^{n}-2 q^{n+2}(q ; q)_{n} \sum_{j=1}^{n} j q^{j-1} \prod_{\substack{k=1 \\ k \neq j}}^{n}\left(1-q^{k}\right) \in \mathbb{Z}[q],
$$

$$
b_{n}(q):=q^{n+1} \sum_{j=1}^{n} q^{j} \prod_{\substack{k=1 \\ k \neq j}}^{n}\left(1-q^{k}\right)^{2} \in \mathbb{Z}[q] .
$$

Theorem 5.1. If $h, k \in \mathbb{N}$, with $\operatorname{gcd}(h, k)=1$, we have

$$
\phi_{1}\left(\frac{h}{k}\right)=8 \pi^{3} i \zeta_{24 k}^{-h}\left(\sum_{n=1}^{k} d_{n}\left(\zeta_{k}^{h}\right)-\sum_{n=1}^{2 k-1} b_{n}\left(\zeta_{k}^{h}\right)\right) .
$$

Remark. Theorem 5.1, together with (1-11) in the case $\tau \in \mathbb{Q} \backslash\{0\}$ of Theorem 1.1, gives an exact value for the integral

$$
\int_{\mathbb{R}} P_{1}\left(w,(-i \tau)^{-1}\right) e^{\pi i \tau w^{2} / 3} \frac{\sinh \left(\frac{2 \pi w}{3}\right)}{\cosh (\pi w)} d w .
$$

To prove Theorem 5.1, we first establish Propositions 5.2 and 5.3 below. These propositions give alternate expressions for the functions defining $\phi_{1}(\tau)$ (see (5-1)), which we subsequently evaluate for $q=\zeta$, where $\zeta$ is any root of unity.

Proposition 5.2. With notation as above, we have

$$
\frac{\partial}{\partial \tau} u_{0}(q)=2 \pi i \sum_{n \geq 1} d_{n}(q) .
$$

Moreover, if $\operatorname{gcd}(h, k)=1$, we have

$$
\frac{\partial}{\partial \tau}\left[u_{0}(q)\right]_{q=\zeta_{k}^{h}}=2 \pi i \sum_{n=1}^{k} d_{n}\left(\zeta_{k}^{h}\right) .
$$

Proof. The first statement follows by straightforward differentiation, using that $u_{0}(q)=\mathcal{U}(0 ; \tau)$, definition (1-2), and the fact that $1 /(2 \pi i)(\partial / \partial \tau)=q(d / d q)$. To prove the second statement, we observe that $d_{n}(q)$ is of the form $d_{n}(q)=$ $(q ; q)_{n-1} \tilde{d}_{n}(q)$, where $\tilde{d}_{n}\left(\zeta_{k}^{h}\right)<\infty$. The statement now follows, observing that for $n \geq k+1$, the factor $(q ; q)_{n-1}$ of $d_{n}(q)$ vanishes when $q=\zeta_{k}^{h}$.

Proposition 5.3. With notation as above, we have

$$
(2 \pi i)^{2} u_{2}(q)=\frac{\partial^{2}}{\partial z^{2}}[\mathcal{U}(z ; \tau)]_{z=0}=-2(2 \pi i)^{2} \sum_{n \geq 1} b_{n}(q) .
$$


Moreover, if $h, k \in \mathbb{N}$, with $\operatorname{gcd}(h, k)=1$, we have

$$
(2 \pi i)^{2} u_{2}\left(\zeta_{k}^{h}\right)=-2(2 \pi i)^{2} \sum_{n=1}^{2 k-1} b_{n}\left(\zeta_{k}^{h}\right) .
$$

Proof. The first statement follows by straightforward differentiation, using definition $(1-2)$, and the fact that $1 /(2 \pi i)(\partial / \partial z)=w(d / d w)$ for $w=e^{2 \pi i z}$. To prove the second statement, using the first statement, we see for $n \geq 2 k$, the $j$-th summand defining $b_{n}(q)$ (for any $j \geq 1$ ) contains either the factor $\left(1-q^{k}\right)$ or $\left(1-q^{2 k}\right)$ (or both), both of which vanish when $q=\zeta_{k}^{h}$.

Proof of Theorem 5.1. Theorem 5.1 now follows from the definition of $\phi_{1}(\tau)$ (see (5-1)), Propositions 5.2 and 5.3.

\section{Acknowledgements}

The authors thank the referee for helpful comments and suggestions. Bringmann's research was supported by the Alfried Krupp Prize for Young University Teachers of the Krupp foundation and the research leading to these results has received funding from the European Research Council under the European Union's Seventh Framework Programme (FP/2007-2013) / ERC Grant agreement n. 335220-AQSER. Folsom is grateful for the support of National Science Foundation CAREER grant DMS-1252815, and the Max Planck Institute for Mathematics in Bonn, Germany.

\section{References}

[Abramowitz and Stegun 1964] M. Abramowitz and I. A. Stegun, Handbook of mathematical functions with formulas, graphs, and mathematical tables, National Bureau of Standards Applied Mathematics Series 55, U.S. Government Printing Office, Washington, DC, 1964. Reprinted by Dover, New York, 1974. MR 29 \#4914 Zbl 0171.38503

[Alfes et al. 2011] C. Alfes, K. Bringmann, and J. Lovejoy, "Automorphic properties of generating functions for generalized odd rank moments and odd Durfee symbols", Math. Proc. Cambridge Philos. Soc. 151:3 (2011), 385-406. MR 2838344 Zbl 1287.11062

[Andrews 2005] G. E. Andrews, "Partitions with short sequences and mock theta functions", Proc. Natl. Acad. Sci. USA 102:13 (2005), 4666-4671. MR 2006a:11131 Zbl 1207.11106

[Andrews 2007] G. E. Andrews, "Partitions, Durfee symbols, and the Atkin-Garvan moments of ranks", Invent. Math. 169:1 (2007), 37-73. MR 2008d:05013 Zbl 1214.11116

[Andrews 2008] G. E. Andrews, "The number of smallest parts in the partitions of $n$ ", J. Reine Angew. Math. 624 (2008), 133-142. MR 2009m:11172 Zbl 1153.11053

[Andrews and Berndt 2009] G. E. Andrews and B. C. Berndt, Ramanujan's lost notebook, Part II, Springer, New York, 2009. MR 2010f:11002 Zbl 1180.11001

[Andrews et al. 2013] G. E. Andrews, R. C. Rhoades, and S. P. Zwegers, "Modularity of the concave composition generating function", Algebra Number Theory 7:9 (2013), 2103-2139. MR 3152010 Zbl 1282.05016 
[Atkin and Garvan 2003] A. O. L. Atkin and F. G. Garvan, "Relations between the ranks and cranks of partitions”, Ramanujan J. 7:1-3 (2003), 343-366. MR 2005e:11131 Zbl 1039.11069

[Atkin and Swinnerton-Dyer 1954] A. O. L. Atkin and H. P. F. Swinnerton-Dyer, "Some properties of partitions", Proc. London Math. Soc. (3) 4 (1954), 84-106. MR 15,685d Zbl 0055.03805

[Bringmann 2008] K. Bringmann, "On the explicit construction of higher deformations of partition statistics”, Duke Math. J. 144:2 (2008), 195-233. MR 2009e:11203 Zbl 1154.11034

[Bringmann and Folsom 2013] K. Bringmann and A. Folsom, "On the asymptotic behavior of Kac-Wakimoto characters", Proc. Amer. Math. Soc. 141:5 (2013), 1567-1576. MR 3020844 Zbl 1277.11035

[Bringmann and Ono 2010] K. Bringmann and K. Ono, "Dyson's ranks and Maass forms", Ann. of Math. (2) 171:1 (2010), 419-449. MR 2011e:11165 Zbl 1277.11096

[Bringmann et al. 2009] K. Bringmann, F. G. Garvan, and K. Mahlburg, "Partition statistics and quasiharmonic Maass forms", Int. Math. Res. Not. 2009:1 (2009), 63-97. MR 2009j:11073 Zbl 1156.11021

[Bringmann et al. 2010] K. Bringmann, J. Lovejoy, and R. Osburn, "Automorphic properties of generating functions for generalized rank moments and Durfee symbols", Int. Math. Res. Not. 2010:2 (2010), 238-260. MR 2011c:11159 Zbl 1230.05034

[Bringmann et al. $\geq 2015]$ K. Bringmann, K. Mahlburg, and R. C. Rhoades, "Peak positions of strongly unimodal sequences", In preparation.

[Bryson et al. 2012] J. Bryson, K. Ono, S. Pitman, and R. C. Rhoades, "Unimodal sequences and quantum and mock modular forms", Proc. Natl. Acad. Sci. USA 109:40 (2012), 16063-16067. MR 2994899

[Cvijović 2009] D. Cvijović, "Closed-form formulae for the derivatives of trigonometric functions at rational multiples of $\pi$ ”, Appl. Math. Lett. 22:6 (2009), 906-909. MR 2010e:33001 Zbl 1231.33001

[Dabholkar et al. 2014] A. Dabholkar, S. Murthy, and D. Zagier, "Quantum black holes, wall crossing, and mock modular forms", preprint, 2014. Submitted for publication. arXiv 1208.4074

[Dyson 1944] F. J. Dyson, "Some guesses in the theory of partitions", Eureka 8 (1944), 10-15. MR 3077150

[Eichler and Zagier 1985] M. Eichler and D. Zagier, The theory of Jacobi forms, Progress in Mathematics 55, Birkhäuser, Boston, 1985. MR 86j:11043 Zbl 0554.10018

[Erdélyi et al. 1981] A. Erdélyi, W. Magnus, F. Oberhettinger, and F. G. Tricomi, Higher transcendental functions, I, Krieger, Melbourne, FL, 1981. Based on notes left by Harry Bateman. MR 84h:33001a Zbl 0051.30303

[Garvan 2011] F. G. Garvan, "Higher order spt-functions", Adv. Math. 228:1 (2011), 241-265. MR 2012g:11185 Zbl 1268.11143

[Kac and Wakimoto 2001] V. G. Kac and M. Wakimoto, "Integrable highest weight modules over affine superalgebras and Appell's function", Comm. Math. Phys. 215:3 (2001), 631-682. MR 2001j:17017 Zbl 0980.17002

[Manschot 2011] J. Manschot, "Wall-crossing of D4-branes using flow trees", Adv. Theor. Math. Phys. 15:1 (2011), 1-42. MR 2888006 Zbl 06074658

[Mellit and Okada 2009] A. Mellit and S. Okada, "Joyce invariants for $K 3$ surfaces and mock theta functions”, Commun. Number Theory Phys. 3:4 (2009), 655-676. MR 2011f:14019 Zbl 1219.14025

[Rademacher 1973] H. Rademacher, Topics in analytic number theory, edited by E. Grosswald et al., Die Grundlehren der mathematischen Wissenschaften 169, Springer, New York, 1973. MR 51 \#358 Zbl 0253.10002 
[Ramanujan 1919] S. Ramanujan, "Some properties of $p(n)$, the number of partitions of $n$ ", Proc. Cambridge Philos. Soc. 19 (1919), 207-210. MR 2280868 JFM 47.0885.01

[Shimura 1973] G. Shimura, "On modular forms of half integral weight", Ann. of Math. (2) 97 (1973), 440-481. MR 48 \#10989 Zbl 0266.10022

[Vafa and Witten 1994] C. Vafa and E. Witten, "A strong coupling test of S-duality", Nuclear Phys. B 431:1-2 (1994), 3-77. MR 95k:81138 Zbl 0964.81522

[Zagier 1991] D. Zagier, "Periods of modular forms and Jacobi theta functions", Invent. Math. 104:3 (1991), 449-465. MR 92e:11052 Zbl 0742.11029

[Zagier 2001] D. Zagier, "Vassiliev invariants and a strange identity related to the Dedekind etafunction”, Topology 40:5 (2001), 945-960. MR 2002g:11055 Zbl 0989.57009

[Zagier 2010] D. Zagier, "Quantum modular forms", pp. 659-675 in Quanta of maths (Paris, 2007), edited by E. Blanchard et al., Clay Math. Proc. 11, Amer. Math. Soc., Providence, RI, 2010. MR 2012a:11066 Zbl 05902011

[Zwegers 2002] S. P. Zwegers, Mock theta functions, thesis, Utrecht University, 2002. Zbl 1194.11058 arXiv 0807.4834

[Zwegers 2010] S. P. Zwegers, "Multivariable Appell functions", preprint, University College Dublin, 2010, Available at http://citeseerx.ist.psu.edu/viewdoc/summary?doi=10.1.1.164.6121.

Received November 13, 2013. Revised April 25, 2014.

KATHRIN BRINGMANN

MATHEMATICAL INSTITUTE

UNIVERSITY OF COLOGNE

WEYERTAL, 86-90

D-50931 KÖLN

GERMANY

kbringma@math.uni-koeln.de

\author{
AMANDA FOLSOM \\ MATHEMATICS \\ YALE UNIVERSITY \\ P.O. BOX 208283 \\ New HaVEn, CT 06520-8283 \\ UNITED STATES \\ amanda.folsom@yale.edu \\ ROBERT C. RHOADES \\ CENTER FOR COMMUNICATIONS RESEARCH \\ 805 BUNN DRIVE \\ PRINCETON, NJ 08540 \\ UNITED STATES \\ rob.rhoades@gmail.com
}




\title{
PACIFIC JOURNAL OF MATHEMATICS
}

\author{
msp.org/pjm
}

Founded in 1951 by E. F. Beckenbach (1906-1982) and F. Wolf (1904-1989)

\section{EDITORS}

Don Blasius (Managing Editor)

Department of Mathematics

University of California

Los Angeles, CA 90095-1555

blasius@math.ucla.edu

\author{
Paul Balmer \\ Department of Mathematics \\ University of California \\ Los Angeles, CA 90095-1555 \\ balmer@math.ucla.edu \\ Robert Finn \\ Department of Mathematics \\ Stanford University \\ Stanford, CA 94305-2125 \\ finn@math.stanford.edu \\ Sorin Popa \\ Department of Mathematics \\ University of California \\ Los Angeles, CA 90095-1555 \\ popa@math.ucla.edu
}

\author{
Vyjayanthi Chari \\ Department of Mathematics \\ University of California \\ Riverside, CA 92521-0135 \\ chari@math.ucr.edu \\ Kefeng Liu \\ Department of Mathematics \\ University of California \\ Los Angeles, CA 90095-1555 \\ liu@math.ucla.edu \\ Jie Qing \\ Department of Mathematics \\ University of California \\ Santa Cruz, CA 95064 \\ qing@ cats.ucsc.edu
}

\section{PRODUCTION}

Silvio Levy, Scientific Editor, production@msp.org

\section{SUPPORTING INSTITUTIONS}

ACADEMIA SINICA, TAIPEI

CALIFORNIA INST. OF TECHNOLOGY

INST. DE MATEMÁTICA PURA E APLICADA

KEIO UNIVERSITY

MATH. SCIENCES RESEARCH INSTITUTE

NEW MEXICO STATE UNIV.

OREGON STATE UNIV.

\author{
STANFORD UNIVERSITY \\ UNIV. OF BRITISH COLUMBIA \\ UNIV. OF CALIFORNIA, BERKELEY \\ UNIV. OF CALIFORNIA, DAVIS \\ UNIV. OF CALIFORNIA, LOS ANGELES \\ UNIV. OF CALIFORNIA, RIVERSIDE \\ UNIV. OF CALIFORNIA, SAN DIEGO \\ UNIV. OF CALIF., SANTA BARBARA
}

\author{
Daryl Cooper \\ Department of Mathematics \\ University of California \\ Santa Barbara, CA 93106-3080 \\ cooper@math.ucsb.edu \\ Jiang-Hua Lu \\ Department of Mathematics \\ The University of Hong Kong \\ Pokfulam Rd., Hong Kong \\ jhlu@maths.hku.hk \\ Paul Yang \\ Department of Mathematics \\ Princeton University \\ Princeton NJ 08544-1000 \\ yang@math.princeton.edu
}

These supporting institutions contribute to the cost of publication of this Journal, but they are not owners or publishers and have no responsibility for its contents or policies.

See inside back cover or msp.org/pjm for submission instructions.

The subscription price for 2015 is US \$420/year for the electronic version, and \$570/year for print and electronic.

Subscriptions, requests for back issues and changes of subscribers address should be sent to Pacific Journal of Mathematics, P.O. Box 4163, Berkeley, CA 94704-0163, U.S.A. The Pacific Journal of Mathematics is indexed by Mathematical Reviews, Zentralblatt MATH, PASCAL CNRS Index, Referativnyi Zhurnal, Current Mathematical Publications and Web of Knowledge (Science Citation Index).

The Pacific Journal of Mathematics (ISSN 0030-8730) at the University of California, c/o Department of Mathematics, 798 Evans Hall \#3840, Berkeley, CA 94720-3840, is published twelve times a year. Periodical rate postage paid at Berkeley, CA 94704, and additional mailing offices. POSTMASTER: send address changes to Pacific Journal of Mathematics, P.O. Box 4163, Berkeley, CA 94704-0163.

PJM peer review and production are managed by EditFLOW ${ }^{\circledR}$ from Mathematical Sciences Publishers.

\section{PUBLISHED BY}

\section{mathematical sciences publishers \\ nonprofit scientific publishing}

http://msp.org/

(C) 2015 Mathematical Sciences Publishers 


\section{PACIFIC JOURNAL OF MATHEMATICS}

Volume $274 \quad$ No. $1 \quad$ March 2015

Unimodal sequences and "strange" functions: a family of quantum modular forms

KATHRIN BRINGMANN, AMANDA FOLSOM and ROBERT C.

RHOADES

Congruence primes for Ikeda lifts and the Ikeda ideal

JIM BROWN and RODNEY KEATON

Constant mean curvature, flux conservation, and symmetry

NiCK EDELEN and BRUCE SOLOMON

The cylindrical contact homology of universally tight sutured contact solid tori

ROMAN GOLOVKO

Uniform boundedness of $S$-units in arithmetic dynamics

Holly Krieger, Aaron LeVin, Zachary Scherr, ThOMAS

TUCKER, Yu YASUFuKu and MiCHAEL E. ZiEVE

A counterexample to the energy identity for sequences of $\alpha$-harmonic 107 maps

YUXIANG LI and YOUDE WANG

Theory of newforms of half-integral weight

Murugesan MANiCKAM, JABAN MEHER and BALAKRISHNAN RAMAKRISHNAN

Algebraic families of hyperelliptic curves violating the Hasse principle

NGUYEN NGOC DONG QUAN

$F$-zips with additional structure

RichaRd PINK, TORSTEN WEDHORN and PAUL ZiEgler

Mean values of $L$-functions over function fields 